\title{
ROTACIÓN MINISTERIAL Y ESTABILIDAD COALICIONAL EN CHILE, 1990-2010
}

\author{
Octavio Avendaño (oavendan@uahurtado.cl) \\ Universidad Alberto Hurtado \\ Mireya Dávila (mireya.davila@iap.uchile.cl) \\ Universidad de Chile
}

\begin{abstract}
Este artículo analiza el fenómeno de la rotación ministerial durante los cuatro gobiernos de la Concertación, que se sucedieron en el período 1990-2010. Junto con describir la participación de los partidos en la conformación de los gabinetes, se examina el impacto de la rotación para la estabilidad del gobierno y para la coalición de centro-izquierda. La rotación ministerial es entendida como el movimiento de ingreso y salida del gabinete ministerial en el marco de una administración gubernamental. En cuanto a la carrera política la rotación se enmarca en la trayectoria que ciertos individuos asumen con el fin de adquirir experiencia, acumular capital político, y desde luego acceder a cargos de representación.
\end{abstract}

Palabras clave: rotación ministerial, Concertación, gabinetes, Chile.

\section{Ministerial Turnover and Coalition Stability IN CHILE, 1990 - 2010}

This paper analyzes ministerial turnover during the four Concertación administrations between 1990 and 2010. In addition to describing party involvement in cabinet formation, we analyze the impact of ministerial turnover on government and coalition stability. Ministerial turnover is defined as the appointment and dismissal of ministers from the cabinet during one administration. In terms of political careers, ministerial turnover is viewed as that aspect of a politician's career trajectory that has the purpose of gaining experience, increasing their political capital and gaining access to elected posts.

Keywords: ministerial rotation, Concertación, cabinets, Chile. 
$\mathrm{E}$ ste artículo analiza el fenómeno de la rotación ministerial durante los cuatros gobiernos de la Concertación de Partidos por la Democracia, que se sucedieron en el período 1990-2010. Junto con describir la participación de los partidos en la conformación de los gabinetes, se analiza el impacto de la rotación para la estabilidad del gobierno y para la coalición de centro-izquierda. La rotación ministerial es entendida como el movimiento de ingreso y salida del gabinete ministerial en el marco de una administración gubernamental. Si se toma en cuenta la carrera política, la rotación se enmarca en la trayectoria que ciertos individuos asumen con el fin de adquirir experiencia, acumular capital político, y desde luego acceder a cargos de representación.

Gran parte de la bibliografía producida a nivel regional ha subrayado la importancia de los gabinetes en el marco de los sistemas presidenciales, y más específicamente de los presidencialismos de coalición. Entre los aspectos que más se destacan sobresale el análisis de la composición interna que presentan los gabinetes (Amorim Neto, 2006a; Dávila, 2011), es decir como variable dependiente; el vínculo con las políticas públicas (Amorim Neto y Borsani, 2003; Altman y Castiglioni, 2009), como variable independiente; la duración de los ministros (Martínez Gallardo, 2011b), y las estrategias presidenciales en el Congreso (Amorim Neto y Samuels, 2010). Por otro lado, las tensiones suscitadas al interior de algunos gobiernos latinoamericanos y europeos han despertado interés en aquellos fenómenos asociados a la estabilidad e inestabilidad de los gabinetes (Martínez Gallardo, 2011). Se trata de una preocupación que se mantiene vigente hasta el día de hoy, debido a las consecuencias políticas que pueden traer consigo tanto las crisis originadas al interior del gabinete como dentro de la propia coalición oficialista.

Respecto a la experiencia chilena, el interés ha estado en la composición de los gabinetes organizados a partir de 1990 y el perfil profesional de los ministros, con la finalidad de analizar el fenómeno de la tecnocracia, o bien para comprender las nuevas formas de liderazgos que surgen desde su interior (Silva, 2010, 2011; Dávila, 2010, 2011; Joignant, 2011). El marcado hincapié puesto en el fenómeno de la tecnocracia se explica, en gran medida, por la importancia que adquieren los técnicos y expertos en las democracias contemporáneas, así como por la conformación de gobiernos con un perfil abiertamente tecnocrático, como ha ocurrido con el de Mario Monti en Italia, en los inicios de la actual administración de Sebastián Piñera, y en algunos gobiernos latinoamericanos que a principios de los noventa aplicaron políticas de ajuste. Pensando en la experiencia chilena de las dos últimas décadas, es posible constatar que el protagonismo de técnicos y expertos adquiere relevancia a 
partir de la política modernizadora impulsada a mediados de los años noventa (Joignant, 2003, 2011), sin desconocer que existen antecedentes que destacan la presencia de tecnócratas en los gabinetes que se sucedieron bajo el régimen militar (Valdés, 1989: Huneeus, 2000) y, con anterioridad, en el período de vigencia del Estado desarrollista (Silva, 2010).

Tomando en cuenta estos antecedentes, resulta primordial entender el significado de la estabilidad ministerial a partir del fenómeno de la rotación, o de los cambios de salida e ingreso entre quienes asumen la titularidad de una cartera ministerial. Existen importantes contribuciones abocadas a estudiar los patrones de cambio ministerial (Martínez Gallardo y Huber, 2003), a través de los cuales es posible explicar la duración de los gabinetes y, al mismo tiempo, conocer la carrera política de los ministros en base a la permanencia y la experiencia acumulada. En esta misma línea, Rodríguez (2009: 10) ha vinculado el fenómeno de la rotación con lo que él denomina acumulación del "capital político" atribuible a quienes asumen diferentes carteras ministeriales a lo largo de un período de tiempo. Existen otros supuestos considerados para este artículo que se relacionan con la política de coalición y la conformación de los gobiernos de coalición. En primer lugar, al estudiar la duración de los ministros en sus cargos es posible conocer la forma en que los partidos miembros de una coalición administran y distribuyen el poder. En segundo lugar, la identificación de los patrones de rotación permite asumir una visión más amplia acerca de la gestión del gobierno y de las políticas públicas. En tercer lugar, el análisis de la trayectoria de la Concertación en el gobierno, en términos de la conformación de sus gabinetes y su permanencia, puede servir de referente para conocer el funcionamiento de otros gobiernos de coalición que existen y han existido en la región.

A estos tres supuestos habría que agregar que la rotación ministerial no necesariamente altera la estabilidad de la coalición y del gobierno. Por el contrario, el cambio de ministros puede ser asumido como un desenlace factible y hasta necesario ante eventuales conflictos, problemas en el desempeño o mala evaluación. En tal sentido, el cambio de ministro da cuenta de una capacidad que adquiere el presidente de la República para nominar a quienes sean considerados política y/o técnicamente adecuados, así como para enfrentar determinadas coyunturas que comprometen tanto al gobierno como a la propia coalición (Martínez Gallardo, 2011). En este artículo se asume como hipótesis que la rotación registrada en los cuatro gobiernos de la Concertación, y que se presenta de manera diferenciada entre cada una de las administraciones, no genera inestabilidad al interior de la coalición. En efecto, la rotación permite atenuar las tensiones que enfrenta el gobierno en ciertas coyunturas, 
en especial cuando se reconocen problemas de desempeño al interior del gabinete. Asimismo, aquellos ministros que experimentan mayor rotación dentro de una misma administración, asumen funciones consideradas claves en gobiernos sucesivos.

En el análisis se considera el grado de incidencia de los partidos en la conformación de los gabinetes durante los gobiernos de la Concertación en el período 1990-2010, los patrones de rotación de las cuatro administraciones y la duración de los ministros en sus cargos. Junto a ello, la rotación se presenta entrelazada con el desarrollo de la carrera política de los ministros, al observar el porcentaje de quienes asumen una nueva cartera u otros cargos públicos habiendo sido titular de una cartera anterior. También analizamos las razones para la salida de los ministros así como el primer destino después de su salida. Respecto de la rotación, y considerando que los cuatro gobiernos de la Concertación presentaron diferencias en la duración del mandato -siendo de cuatro años los gobiernos de Patricio Aylwin (1990-1994) y Michelle Bachelet (2006-2010) y de seis en los de Eduardo Frei R.-T. (1994-2000) y Ricardo Lagos (2000-2006)-, se procedió a dividir el número de meses de cada administración por el número de ministros. El artículo ha sido estructurado en cuatro secciones. La primera contiene aspectos teóricos que dan cuenta de la relevancia alcanzada por los gabinetes al interior de los gobiernos de coalición, en los presidencialismos latinoamericanos. La segunda, aborda la relación entre carrera política y rotación ministerial. La tercera da cuenta de los hallazgos empíricos de la investigación, cuyos datos son presentados comparando lo que ocurre en cada una de las administraciones. La cuarta presenta las principales conclusiones de este trabajo.

\section{Los gabinetes en los gobiernos de los presidencialismos de coalición}

De acuerdo a la definición de Müller y Strøm (2000:11), los gabinetes representan "el conjunto de agentes del ejecutivo designados políticamente en el nivel más alto del sistema de policy making"; constituyen, además, un ámbito de gobierno en el que se definen buena parte de las políticas que implementan los gobiernos en las democracias contemporáneas. Los gabinetes permiten vincular a los partidos con el gobierno, debido a las discusiones parlamentarias que generan las iniciativas impulsadas por el Ejecutivo. Las variaciones y los criterios en la designación de quienes integran cada gabinete permiten explicar las diferencias en policy making, en términos de representación de intereses en las democracias (Amorim Neto, 2006; Amorim Neto y Samuels, 2010), así como los conflictos entre los intereses propiamente políticos y los de la administración (Botella y Rodríguez, 2005). Es posible también entender 
las designaciones ministeriales y su permanencia como un espacio de cooperación o disputa dentro de la propia coalición de gobierno, en la medida que el presidente satisfaga las demandas partidarias al respecto.

Sobre coaliciones y gabinetes en sistemas parlamentarios existen importantes aportes en el plano teórico como empírico (Gamson, 1961; Riker, 1962; Laver, 2000). A la hora de definir a las coaliciones, éstas pasan a ser concebidas como acuerdos políticos centrados en la cooperación. De acuerdo a lo afirmado por Strøm (1990), la formación de coaliciones facilita las negociaciones y acuerdos entre los partidos y, especialmente, entre el presidente y los partidos que integran el gabinete. Esto pasa por establecer un acuerdo programático, el respaldo de los partidos en el parlamento y la posibilidad de acceder a los principales cargos públicos por parte de quienes forman parte del gobierno.

En relación a los sistemas presidenciales, la literatura sobre coaliciones y gabinetes es mucho más reciente, pese a que, como señala Shugart y Samuels (2009), desde fines del siglo XX la mayoría de las democracias tienen presidentes elegidos directamente. Gran parte de los trabajos dedicados a analizar el funcionamiento de las democracias presidencialistas latinoamericanas vinculan la formación de los gabinetes a la existencia de coaliciones partidarias, que se han venido imponiendo en varios gobiernos de la región (Amorim Neto, 1998; Lanzaro, 2003; Chasquetti, 2008; Camerlo, 2012). Se trata de un tipo de presidencialismo que presenta una serie de atributos que lo distinguen del presidencialismo clásico, que tendía a la formación de gobiernos de minoría y que enfrentaba permanentes crisis de gobernabilidad. El "nuevo presidencialismo de coalición" se sustentaría en la presencia de gobiernos que reciben un respaldo amplio por parte de la población, fomenta la colaboración y el acuerdo entre los partidos integrantes de la coalición, y fomenta la formación de nuevos actores con veto, normalmente vinculados a los partidos del oficialismo.

Con la implementación de reformas y ajustes estructurales, que se llevaron a cabo desde fines de los años ochenta, se vieron fortalecidas las atribuciones de los poderes ejecutivos de varios países de la región (Murillo, 2001; Stokes, 2001). En algunos casos, el aumento de las atribuciones del ejecutivo y el carácter decisionista de la gestión presidencial derivó en situaciones de crisis (Pérez Liñán, 2009), o en el desplazamiento de los partidos en el gobierno por oposiciones que canalizaron el descontento generado por medidas tales como la disminución del gasto público y la privatización de las empresas del Estado. Sin embargo, la inestabilidad y la crisis política que acompañó a este tipo de iniciativas logró ser superada mediante la formación de alianzas y coaliciones que aseguraron un mayor respaldo legislativo a la gestión gubernamental. 
Esto último ha sido corroborado por Altman y Castiglioni (2009) quienes, al analizar las consecuencias políticas de las reformas estructurales implementadas en América Latina, demuestran que mientras más partidista era un gabinete, menor era el impacto de ese tipo de medidas ${ }^{1}$. Según Altman y Castiglioni, un gabinete compuesto mayoritariamente de técnicos está menos preocupado de los costos políticos que pueden acarrear determinadas iniciativas y reformas, que uno compuesto por representantes y miembros activos de los partidos que sustentan y respaldan al gobierno (Altman y Castiglioni, 2009: 20). Adicionalmente, constatan que la presencia de gobiernos de coalición también ha facilitado la implementación de reformas estructurales, sobre todo cuando desde los partidos de una coalición oficialista se promueven los ministros y los encargados de ejecutar tales políticas. Una situación muy distinta experimentan aquellos presidentes que no negocian con actores partidarios y dejan la política pública en manos de tecnócratas.

Otros autores han puesto hincapié en aspectos de las estrategias legislativas del presidente y la influencia sobre determinadas políticas. Autores como Amorim Neto $(1998,2006)$ y Amorim Neto y Samuels (2010) han analizado la relación entre las estrategias presidenciales de policy making y los patrones de asignación del gabinete. Además, establecen una tipología de gabinetes dependiendo de si los presidentes designan militantes, independientes, o bien según su estrategia legislativa. De acuerdo a la constatación de estos autores, en sistemas presidenciales los gabinetes no siguen necesariamente la proporcionalidad dado que su formación tiene un origen menos colectivo que en sistemas parlamentarios. Adicionalmente, Amorim Neto y Strøm (2006) y Amorim Neto y Samuels (2010) sostienen que los gabinetes han tendido a diminuir la presencia de miembros de partido, debido a la mayor presencia de independientes, y a tener una conformación más proporcional en democracias presidenciales que en las parlamentarias.

En el caso de Chile, los estudios de los gabinetes que se conformaron a partir de 1990 se pueden dividir en indirectos y directos. Los estudios indirectos se refieren a los gabinetes en función de su relación con el sistema político y sobre todo con el diseño institucional creado en la Constitución de 1980 (Garretón, 1992, 1996;

1 Se podría decir que ocurriría una situación más o menos análoga a la de aquellos gobiernos que contaron, para el mismo fin, con una fuerte base partidaria y de arraigo social, como ocurrió en la argentina menemista de la primera mitad de los años noventa (Murillo, 2001; Stokes, 2001; Levistky, 2005). 
Siavelis, 1997, 2000; Huneeus, 2005)2. Por el contrario, los estudios directos que se centran exclusivamente en la conformación del gabinete (Amorim Neto, 1998, 2006; Garrido, 2003; Altman, 2008; Martínez Gallardo, 2011). La preocupación por la conformación de los gabinetes incluye, en muchos casos, el análisis acerca de las elites, la distribución partidaria, el liderazgo del presidente, y finalmente las dinámicas intra-coalición como factor explicativo de su estabilidad interna y de la propia coalición de gobierno. Sobre esto último, Dávila (2011) desarrolla un análisis de los factores intra coalición que ayudaron a la cooperación de los partidos miembros de la Concertación; analizando la estabilidad de los gabinetes como medida de estabilidad, la autora argumenta que una mayor autonomía del presidente respecto de los partidos, el uso de reglas informales de distribución del poder y el uso político de expertos (en este caso tecnócratas), ayudan a extender el buen resultado de la coalición en términos de gobernabilidad. Además, la autora avanza al analizar el peso de las reglas informales en las cuatro administraciones concertacionistas.

\section{Carrera política y rotación ministerial}

Dentro de los estudios más importantes aparecidos en el último tiempo, y que dan cuenta de la composición y la función de los gabinetes, se observa un notorio énfasis en el fenómeno de la carrera política de los ministros (por ejemplo, Rodríguez, 2009). Tanto para los ministros como para el conjunto de líderes que surgen en las democracias contemporáneas, la carrera política es asumida en términos de tendencia hacia la profesionalización. A partir del ensayo de Max Weber La política como vocación (1996), existe un reconocimiento explícito de la importancia que adquiere el político profesional, el cual se desenvuelve en diversos ámbitos de representación y deliberación. En la visión de Weber, el político profesional, que se convierte en figura central dentro y fuera de los partidos, tiene su origen en aquellos funcionarios que en los inicios del Estado moderno colaboraban directamente con los príncipes y gobernantes. En la medida en que las organizaciones partidarias se van dotando de individuos que viven "de" la política y no sólo "para” la política, ésta va adquiriendo el carácter de actividad permanente.

2 Para otras épocas históricas, el estudio de los gabinetes también ha sido mayoritariamente indirecto. En efecto, se los ha mencionado como parte de otros aspectos del sistema político, como puede ser el análisis sobre el sistema de partidos, y sobre todo, las relaciones Ejecutivo - Legislativo. Así por ejemplo, la literatura que ha analizado el quiebre de la democracia en Chile ha demostrado que la configuracion de la relación entre el Ejecutivo y Legislativo ha sido definitoria en determinar los incentivos para la cooperación entre las elites (Valenzuela, 1978; Siavelis, 1997). 
En la actualidad la presencia de políticos profesionales es concebida como una expresión de gobiernos responsables y de rendición de cuenta (accountability) efectiva. Como ha escrito Botero: "Es deseable tener políticos profesionales que son más responsables frente a las demandas de los electores que aquellos políticos que ocupan su cargo y después desaparecen" (Botero, 2011: 171). Se trata, por cierto, de una aseveración que es válida para quienes ocupan cargos electos, o que se desenvuelven en las instituciones representativas. De todos modos, sería extrapolable a quienes han ocupado carteras ministeriales y cuya carrera se proyecta hacia los cargos de elección popular.

Sobre el tema de la carrera política de los ministros, existe una importante bibliografia teórica y empírica abocada a los gabinetes de distintos países europeos. La mayoría de los trabajos analiza el tema de los gabinetes desde la mirada de los actores y sus características en democracias parlamentarias. Entre los trabajos más importantes sobresalen aquellos derivados de comparaciones sobre distintos países (Dogan, 1981; Blondel, 2001; Blondel, Müller-Rommel y Malová, 2007; Tavares de Almeida, Costa Pinto y Bermeo, 2003; Botella, Rodríguez, Barberá, Barrio, 2011), aunque también es importante la contribución que realizan determinados estudios de caso (Buckley, 2006; Andeweg y Timmermans, 2008; Back et al., 2009). En sistemas presidenciales, la literatura que analiza los gabinetes desde la perspectiva individual de los ministros tiende a ser mucho más escasa, con la excepción de los estudios de Ai Camp (1995, 2002), que analizan el caso mexicano en función de las trayectorias de las elites de gobierno.

Otra de las aproximaciones proviene del efecto que significa la presencia de tecnócratas y expertos en el gobierno y en las instancias en las cuales se define la política pública. La presencia de gobiernos con un perfil estrictamente técnico se reconoce en contextos en los cuales se ha experimentado un proceso de crisis del sistema de partidos, como ha ocurrido en países europeos y también latinoamericanos. En países como Brasil, Argentina y Perú, a inicios de los años noventa la organización de gobiernos con perfil técnico, y la presencia de técnicos en las principales decisiones del gobierno, estuvo relacionada con el discurso antipolítico y la implementación de políticas de ajuste (Stokes, 2001). Distinta sería la interpretación que hace Alcántara sobre el predominio de la tecnocracia, según él: “La aparición de 'élites expertas', profundamente desideologizadas, es un fenómeno novedoso al que de alguna manera se ha llegado por la propia profesionalización de la política” (Alcántara, 2012: 22).

Para el caso de Chile, Joignant (2011) analiza los diferentes conocimientos o saberes de la elite concertacionista y su ubicación en los gabinetes de los cuatro gobiernos de la Concertación. Por su parte, De La Maza (2011) analiza la trayectoria desde el mundo civil al gobierno entre 1990 y 2006 a través del estudio de los ministerios políticos y 
de Mideplan. El predominio de la tecnocracia y de profesionales altamente calificados al interior de los gabinetes puede resultar, más bien, un fenómeno circunstancial que una tendencia que se impone de manera definitiva. Incluso, podría llegar a cambiar el tipo de experticia requerida para asumir determinados cargos, como ocurre con la creciente importancia que se le asigna al conocimiento económico en la toma de decisiones.

La carrera y la experiencia acumulada por los ministros constituyen una dimensión central para el estudio del funcionamiento de los gabinetes. Se parte del supuesto que la experiencia de un ministro no sólo implica mayores conocimientos y habilidades técnicas; también implica la asimilación de un conjunto de destrezas y habilidades políticas. Adicionalmente, el estudio de la carrera de los ministros permite entender el nivel de rotación y de estabilidad al interior del gobierno en un período específico de tiempo (Camerlo, 2012). La carrera implica asumir por un lado un conjunto de antecedentes correspondientes al momento previo a la designación del cargo de ministro. También implica tomar en cuenta el conjunto de cargos que puede asumir dentro del gobierno, una vez abandonado el cargo de ministro. A juicio de Blondel y Thiébault (1991), la carrera ministerial comprende aquel período de la trayectoria de un individuo que transcurre entre el primer nombramiento ministerial y la última salida del gobierno.

Este tipo de definiciones conllevan un importante problema de tipo metodológico. ¿Desde cuándo es posible analizar la carrera si muchos de los ministros cuentan con antecedentes y experiencias políticas o técnicas adquiridas en una fase previa a la de la nominación? Al respecto, Rodríguez (2009: 6) plantea la necesidad de considerar aquellos cargos desempeñados tanto en el ámbito público, en el privado, en el de las organizaciones sociales y grupos de interés. Borrelli et al. (2012) han indagado en el tema de la decisión de los ministros de abandonar el cargo, ya sea para salir definitivamente del gobierno o para asumir otras responsabilidades dentro del mismo. En el desarrollo de la carrera política es fundamental la rotación en diferentes carteras, debido al efecto que eso tiene en términos de influencia y de experiencia política.

En la experiencia de los gobiernos de la Concertación, el paso de un ministerio a otro, intercalado con una subsecretaría, directorio de empresa pública y a veces con una misión de tipo diplomático, se da de manera recurrente en los gabinetes de las administraciones que se suceden desde 1994 en adelante. A su vez, este paso se va transformando en una carrera en sí misma. Mientras más cargos de ministro se acumulan en un solo individuo, mayor experiencia y capacidad de intervención en momentos y coyunturas de crisis, además de poder ocupar posiciones estratégicas al interior del gobierno. 


\section{Gabinetes y rotación ministerial en el período 1990-2010}

Según se ha definido en este trabajo, la rotación es entendida como el cambio que experimenta un ministro dentro del gabinete. Dicho cambio no solo implica el abandono definitivo del gabinete, como va ocurrir con varios ministros a partir de 1994, sino que en muchos casos significará el paso de una cartera a otra. Por otro lado, la salida de un ministro no necesariamente da cuenta de situaciones de tensión y de crisis al interior del gobierno, o de la coalición que lo sustenta. Como lo demuestra la experiencia chilena en el período estudiado, la salida de algunos ministros se enmarca dentro de una estrategia definida por el propio gobierno, siendo además coherente con el desarrollo de la carrera política de quienes ocupan determinadas carteras. Para llevar a cabo el análisis de la rotación ministerial, se calcula, en primer lugar, la duración en el puesto de cada ministro; posteriormente se establecen intervalos de duración a fin de agruparlos y proceder a comparar lo que ocurre durante las cuatro administraciones de la Concertación.

\section{Los ministros y los partidos}

Lo primero que caracteriza al conjunto del período comprendido por los cuatro gobiernos de la Concertación es la fuerte presencia de los partidos en la composición de los gabinetes. Tal como se muestra en la Tabla 1, de los 169 ministros que integraron los gabinetes de las cuatro administraciones de la coalición de centroizquierda, la presencia de ministros sin filiación partidaria fue más bien marginal, dado que estuvieron por debajo el 6,5\%.

Tabla 1

Militancia de los ministros de la Concertación (1990 - 2010). Número total y porcentajes

\begin{tabular}{|l|c|c|}
\hline \multicolumn{1}{|c|}{ Partido } & Número de Ministros & Porcentaje del Total (\%) \\
\hline PDC & 77 & 45,5 \\
\hline PS & 37 & 21,9 \\
\hline PPD & 33 & 19,5 \\
\hline PR & 10 & 5,9 \\
\hline PAC & 1 & 0,6 \\
\hline Independientes & 11 & 6,5 \\
\hline Total & 169 & 100 \\
\hline
\end{tabular}

Fuente: Elaboración propia 
El desglose de la participación de los partidos en cada una de las administraciones, que se observa en el gráfico 1, muestra que la mayor presencia de ministros independientes y sin filiación partidaria se produjo durante el gobierno de Patricio Aylwin. A medida que se suceden las otras administraciones de la Concertación, la presencia de ministros independientes disminuye. Llama la atención la fuerte presencia de ministros con militancia partidaria dado el énfasis puesto en el tema de la modernización y la inserción en los mercados internacionales durante el gobierno de Frei, la reactivación económica durante el gobierno de Lagos y la creación de un "gobierno ciudadano" bajo la administración de Bachelet (Joignant, 2003; Mardones, 2007; Morales, 2008).

Gráfico 1

Militancia de los ministros en cada administración 1990-2010

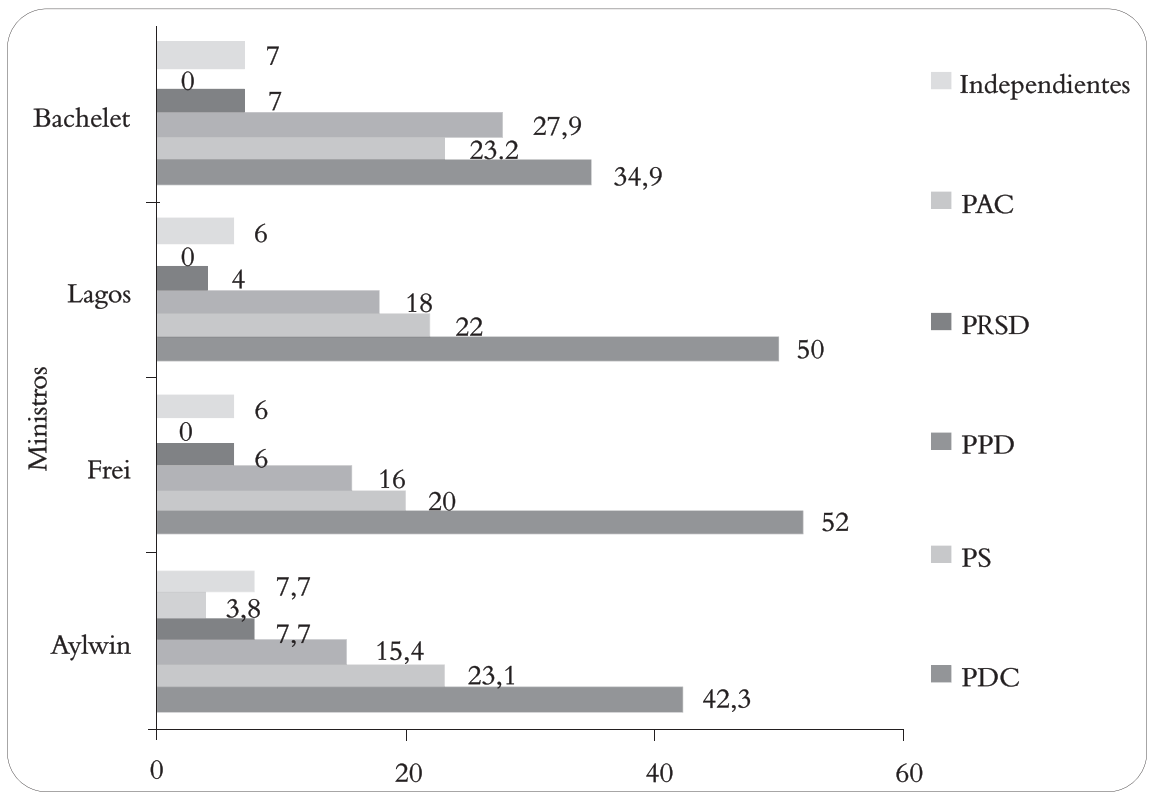

Fuente: Elaboración propia

Lo que ocurre al interior de los gabinetes durante los gobiernos de la Concertación refleja una tendencia contraria respecto a lo sucedido en otras instancias en las cuales, efectivamente, los partidos fueron perdiendo protagonismo y poder de incidencia. En gran medida, el debilitamiento que sufrieron los partidos puede ser entendido por la importancia asignada a los grupos de asesores y a las comisiones 
de expertos durante los gobiernos de Ricardo Lagos y, sobre todo, de Michelle Bachelet (Silva, 2011). Si se consideran los objetivos definidos para el gobierno de Eduardo Frei, la presencia mayoritaria de ministros con filiación partidaria permite asegurar el respaldo para la implementación de políticas destinadas a acelerar la modernización. En tal sentido, se corrobora la tesis de Altman y Castiglioni (2009) sobre la importancia que tienen los gabinetes partidarios para atenuar los efectos de las políticas de modernización y de ajuste implementadas en otros países de la región.

La información del gráfico 1 refleja también una mayor preponderancia del PDC en la distribución de los cargos ministeriales. De los cuatro gobiernos de la Concertación, el de Aylwin tendió a generar mayores equilibrios entre los partidos de la coalición al nominar los titulares de cada ministerio. En ese mismo gobierno, el porcentaje de ministros del eje PS-PPD fue similar al del PDC. A diferencia de las tres primeras administraciones, durante el gobierno de Bachelet, el eje PS-PPD superó con creces la presencia de ministros ligados al PDC, siendo de $51,1 \%$ y $34,9 \%$ respectivamente.

Tabla 2

Nivel de proporcionalidad entre el porcentaje de respaldo electoral y de participación en el gabinete

\begin{tabular}{|l|c|c|c|c|c|}
\hline & PDC & PS & PPD & PR & PAC \\
\hline Aylwin & $-7,8$ & 2,2 & 0 & 1,2 & 5,3 \\
\hline Frei & $-1,9$ & $-3,9$ & 0 & 0,3 & 0 \\
\hline Lagos & $-5,7$ & 1,5 & 0 & 0 & 0 \\
\hline Bachelet & $-2,7$ & $-2,0$ & 0 & $-7,0$ & 0 \\
\hline
\end{tabular}

Fuente: Elaboración propia

En la distribución de los ministerios la búsqueda de equilibrios se asume en función de la coalescencia, o proporcionalidad, entre el número de cargos asignados a un partido con aquellos que posee al interior del Congreso Nacional. Dicha relación ha sido largamente estudiada en los sistemas parlamentarios (Browne y Franklin, 1973). En cambio, en el marco de los sistemas presidenciales, y sobre todo en los presidencialismos de coalición, se depende de los equilibrios partidarios que asuma el propio presidente. En la experiencia de las cuatro administraciones de la Concertación, la proporcionalidad fue aplicada por el presidente considerando no sólo el peso electoral de los partidos sino también la necesidad de mantener la estabilidad de la coalición (Dávila, 2011). Por ende, y tal como aparece en la tabla 2, hubo partidos, 
como el PDC que quedaron subrepresentados en los gobiernos de Aylwin y Lagos, mientras que en el primer gobierno de la Concertación una de las organizaciones claramente sobrerrepresentadas fue el Partido Alianza de Centro (PAC).

Además de la notoria presencia de ministros partidistas, habría que destacar una diferencia importante a nivel de los ministros de las administraciones que se sucedieron entre una década y otra. En la primera década, correspondiente a los gobiernos de Aylwin y de Frei, fue notoria la presencia de los principales dirigentes de los partidos al interior de los gabinetes que se formaron en ambas administraciones. Durante el gobierno de Aylwin destacó la presencia de importantes dirigentes, como Enrique Silva Cimma, Ricardo Lagos, Jorge Arrate, Edgardo Boeninger y Germán Correa. Algo similar ocurre en el gobierno de Frei debido a la presencia de Germán Correa, Jorge Arrate, Ricardo Lagos, Soledad Alvear y Edmundo Pérez Yoma, quienes jugaron roles claves dentro de sus respectivos partidos y dentro de la propia coalición. La presencia de ministros con altos cargos y funciones partidarias decae completamente en la década siguiente. Sin embargo, en los dos últimos gobiernos se observa la presencia de ministros cuyo capital político fue acumulado a raíz de la sucesiva participación en los gabinetes que fueron organizados, como ocurrió con figuras tales como José Miguel Insulza, Edmundo Pérez Yoma, Sergio Bitar y Francisco Vidal.

\section{Rotación y estabilidad ministerial}

Para analizar el fenómeno de la rotación ministerial se procedió al establecimiento de intervalos, que fueron de 0 a 5 en función de los meses de duración. El intervalo 0 da cuenta de un período de duración que va entre 0 y 6 meses; luego el 1 que comprende desde 6,1 a 12 meses; el 2 de 12,1 a 24 meses; el 3 de 24,1 a 36 meses; el 4 que indica más de 36 meses y el 5 todo el período de gobierno, que en las administraciones de Aylwin y Bachelet comprendió 48 meses, mientras que en las de Frei y Lagos fue de 72. Como se observa en la tabla 3, el gobierno con menor rotación ministerial fue el de Aylwin. El resto de los gobiernos se caracterizó por una mayor rotación. En el caso particular de los gobiernos de Frei y Bachelet, hubo una alta rotación en los primeros doce meses. Del total de ministros para cada administración, el 34\% en la de Frei y el 25,6\% en Bachelet se mantuvo durante un período inferior a doce meses. Durante el gobierno de Aylwin la mitad de sus ministros permanecieron todo el período, lo cual se explica por el desafío que existía para dicho gobierno de tener buen desempeño y garantizar condiciones de gobernabilidad. 
Tabla 3

Rotación Ministerial en Gobiernos de la Concertación (1990 - 2010) (en porcentaje)

\begin{tabular}{|l|c|c|c|c|}
\hline Intervalo & Aylwin & Frei & Lagos & Bachelet \\
\hline 0 & 3,8 & 16,0 & 2,0 & 11,6 \\
\hline 1 & 0 & 18,0 & 14,0 & 14,0 \\
\hline 2 & 19,2 & 14,0 & 42,0 & 32,6 \\
\hline 3 & 19,2 & 18,0 & 16,0 & 30,2 \\
\hline 4 & 7,7 & 32,0 & 20,0 & 7,0 \\
\hline 5 & 50,0 & 2,0 & 4,0 & 4,7 \\
\hline
\end{tabular}

Fuente: Elaboración propia

Tanto en los gobiernos de Lagos y Bachelet, se dio la coincidencia de que fueron los ministros de Hacienda respectivos -Nicolás Eyzaguirre y Andrés Velasco- quienes permanecieron por todo el período. Durante la administración de Eduardo Frei, su Ministro de Hacienda Eduardo Aninat dejó el cargo en diciembre de 1999, es decir, meses antes de que culminara el período constitucional de dicha administración, para asumir un cargo en un organismo internacional. Esto permite corroborar la importancia que, desde un comienzo, tuvo para la Concertación la necesidad de generar condiciones de estabilidad macroeconómica (Boeninger, 1998; Hidalgo, 2011; Varas, 2012).

Tabla 4

Número de Rotaciones por ministerio y administración ${ }^{3}$ (1990 - 2010)

\begin{tabular}{|c|c|c|c|c|}
\hline \multirow{2}{*}{ Gobierno } & \multicolumn{4}{|c|}{ Número de rotaciones } \\
\cline { 2 - 5 } & 3 & 2 & 1 & 0 \\
\hline Aylwin & 0 & 1 & 3 & 18 \\
\hline Frei & 3 & 6 & 6 & 1 \\
\hline Lagos & 3 & 7 & 12 & 3 \\
\hline Bachelet & 0 & 5 & & 2 \\
\hline
\end{tabular}

Fuente: elaboración propia

3 Número de ministerios en que sus ministros no han rotado $(0)$, han cambiado una vez en el período (1), 2 y 3 veces. 
Complementando lo presentado anteriormente, la tabla 4 permite observar diferencias en la rotación ministerial de los gobiernos que se sucedieron a partir de 1994. La administración Frei tuvo un mayor porcentaje de ministros que duraron más de tres años (el 32\%), mientras que en las de Lagos y Bachelet, los ministros duraron mayoritariamente entre un año y dos (42\% y 32,6\% respectivamente). Al relacionar los ministerios con el número de rotaciones, se observa un patrón de estabilidad y de escasa rotación en el Ministerio de Hacienda. Los gobiernos no cambiaron el ministro de Hacienda salvo el presidente Frei, por la salida de Aninat mencionada anteriormente. En el otro extremo, el Ministerio de Educación fue el que presentó el mayor número de rotaciones en las cuatro administraciones concertacionistas. Salvo el gobierno de Aylwin, en el que se sucedieron dos ministros, equivalentes a una rotación ministerial, el resto de los gobiernos tuvo entre tres y cuatro rotaciones en dicha cartera.

\section{Las trayectorias de los ministros, las salidas y sus destinos}

Del total de ministros que existieron durante los cuatro gobiernos de la Concertación, (169), 23 tuvieron dos o más cargos de ministros. En términos porcentuales, esto quiere decir que sólo un $13,4 \%$ de los ministros desarrollaron una carrera ministerial en ese período.

Tabla 5

Ministros con dos o más cargos

\begin{tabular}{|c|c|}
\hline Número de cargos & Ministros \\
\hline Con 2 Cargos & 18 \\
\hline Con 3 cargos & 4 \\
\hline Con 4 cargos & 1 \\
\hline
\end{tabular}

Fuente: elaboración propia

Dada la importancia que tiene la formación de coaliciones en sistemas presidenciales, se hace necesario analizar las causas de salida y el destino inmediato de los ministros una vez abandonada la cartera respectiva. En una coalición que estuvo veinte años en el poder, es interesante conocer y explicar las trayectorias de los ministros más allá de sus cargos. En la siguiente tabla presentamos una tipología de causas de salida de los ministros de la Concertación. Según lo reflejado en la tabla 6, durante la administración Aylwin no hubo, al menos aparentemente, problemas en temas de 
corrupción, ni de manejo político y apenas un caso de rotación se debió a problemas de manejo de la política, como ocurrió en ese entonces con el Ministro de Salud después de enfrentar un paro de los médicos. Esto no implica que no haya habido problemas de manejo en el gobierno, sino más bien que se optó por mantener lo más intacto posible el primer gabinete como expresión de estabilidad (Dávila, 2011).

Tabla 6

Causas de salida de los ministros de la Concertación (1990 - 2010)

\begin{tabular}{|l|c|c|c|c|}
\hline \multicolumn{1}{|c|}{ Causa } & Aylwin & Frei & Lagos & Bachelet \\
\hline Personal & 1 & 7 & $8(32 \%)$ & 2 \\
\hline Elecciones & $4(66,6 \%)$ & 4 & 6 & 4 \\
\hline $\begin{array}{l}\text { Corrupción/ } \\
\text { escándalo }\end{array}$ & 0 & 3 & 1 & 0 \\
\hline Manejo político & 1 & 14 & 9 & 14 \\
\hline Otros & 0 & 2 & 1 & 2 \\
\hline Total & 6 & 30 & 25 & 22 \\
\hline
\end{tabular}

Fuente elaboración propia

Por otro lado, si se observan las causas de salida de los ministros en las tres administraciones restantes, no se identifica un patrón a destacar. Sin embargo, es posible mencionar que en el caso de la administración Lagos, el mayor porcentaje de causas de rotación de los ministros es la denominada personal, es decir tuvo que ver con decisiones del presidente frente a los miembros de la coalición, las que se expresan en nuevas nominaciones, cambios y traslados de un cargo a otro. Los gobiernos de Frei y Bachelet coinciden en que el mayor porcentaje de causas de salida son problemas de manejo de políticas vinculadas a los ministerios. Se observa que el porcentaje de salida de ministros a causa de las elecciones es similar en las cuatro administraciones. 


\section{Gráfico 2}

Actividades de los ministros después del ministerio. Período 1990-2006

(en porcentaje)

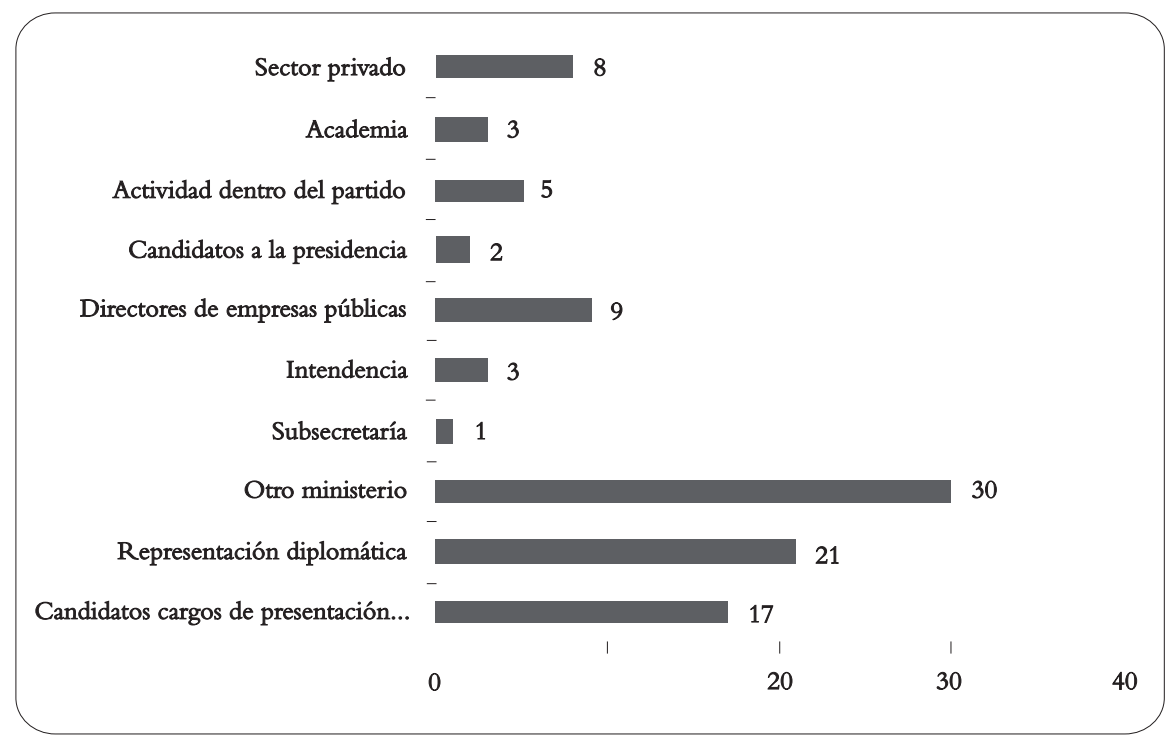

Fuente elaboración propia

Finalmente, al observar lo que ocurre después de que un Ministro deja su cargo, tal como aparece en el gráfico anterior, se constata que además de que muchos vuelven a ser ministros, gran parte siguen ligados al sector público, a cargos de representación popular, o en otras funciones encomendadas por el Ejecutivo. Este hecho es sintomático al observar lo que ocurre con la trayectoria posterior de aquellas personas que dejan el ministerio en el período 1990 a 2006. Se toma sólo ese período ya que corresponde a una etapa en la cual la Concertación puede proyectar la distribución de cargos públicos no electivos entre personeros de sus filas, o bien con sectores independientes cercanos a ella.

\section{Conclusiones}

Uno de los rasgos más sobresalientes de las cuatro administraciones de la Concertación fue el marcado carácter partidista de sus respectivos gabinetes, lo que refleja el nivel de incidencia que conservaron los partidos en la formación de los gobiernos 
que se sucedieron a partir de 1990. Contrariando la tendencia hacia un estilo estrictamente tecnocrático, la presencia de los partidos se mantiene e incluso aumenta en los gobiernos de Frei y de Lagos. La presencia de ministros independientes, o sin filiación partidaria, puede ser considerada mínima y en algunos momentos marginal. Cabe agregar algo que ya ha sido constatado por otros estudios (Dávila, 2010, 2011), que los ministros que participaron en carteras consideradas técnicas, la mayoría de ellos posee filiación partidaria.

Otro hecho que deviene significativo, a nivel de lo que se ha denominado la rotación ministerial, es el paso que se produce de un ministerio a otro o bien desde un ministerio a otro tipo de cargos públicos, como pueden ser alguna subsecretaría, directorio de empresa pública y a veces con una misión de tipo diplomático, se da de modo recurrente en los gabinetes de los gobiernos que se suceden desde 1994 en adelante. A su vez, este paso se va transformando en una carrera en sí misma. Mientras más cargos de ministro se acumulan, mayor capacidad de intervención tendrá dicho individuo en momentos y coyunturas de crisis, o de ocupar posiciones estratégicas al interior del gobierno. Es el caso de figuras como José Miguel Insulza, Francisco Vidal, Edmundo Pérez Yoma, Sergio Bitar, y en menor medida de Belisario Velasco, quien construye toda una trayectoria en su calidad de subsecretario, pero que juega un rol clave como Ministro del Interior bajo el gobierno de Bachelet.

El hecho de participar en varias ocasiones en calidad de Ministro redunda también en proyección política y en la emergencia de nuevos liderazgos. Esta proyección se expresa a escala local pero también a nivel internacional, pensando en figuras como José Miguel Insulza y su labor al interior de la OEA. Paradójicamente, los ministros que han tenido mayor proyección política son aquellos que provienen de carteras ministeriales más técnicas, y no necesariamente quienes se han desempeñado en los ministerios sociales -como Mideplan, Educación o Trabajo-, o en aquellos estrictamente políticos: Interior, Secretaría General de Gobierno, Secretaría General de la Presidencia. Si se piensa en la experiencia y la mayor visibilidad alcanzada por Ricardo Lagos en el Ministerio de Obras Pública, de Michelle Bachelet en Defensa, del propio Andrés Velasco, como el flamante y controvertido Ministro de Hacienda de Michelle Bachelet. Desde el punto de vista institucional, los ministerios que presentaron más rotación fueron los de Defensa y Educación. La explicación posible se podía encontrar en la complejidad de las relaciones cívico-militares en contextos de democratización en el caso de Defensa, y el poder de los gremios u otros grupos de interés en lo que respecta a Educación. 


\section{Referencias Bibliográficas}

Alcántara, Manuel (2012). El oficio del político. Madrid: Editorial Tecnos.

Altman, David (2008). "Political Recruitment and Candidate Selection in Chile (1990-2003): The Executive Branch". En Scott Morgenstern y Peter Siavelis (Eds.). Pathway to Power in Latin America. University Park: Penn State University Press.

Altman, David y Rossana Castiglioni (2009). "Gabinetes ministeriales y reformas estructurales en América Latina, 1985-2000”. Revista Uruguaya de Ciencia Política,Vol. 18, № 1, pp. 15-39.

Ai Camp, Roderic (1995). Political recruitment across two centuries. México, 1884 - 1991. Austin, TX. University of Texas Press.

Ai Camp, Roderic (2002). Mexico's Mandarins. Crafting a Power Elite for the Twenty-first Century. Berkeley, CA. University of California Press.

Amorim Neto, O. \& Samuels, D. (2011). "Democratic Regimes and Cabinet Politics: a Global Perspective". Revista Ibero-Americana de Estudos, Vol 1, № 1, pp. 10-23.

Amorim Neto, O. \& Borsani, H. (2004). "Presidents and cabinets:The political determinants of fiscal behavior in Latin America". Studies in Comparative International Development, Vol. $39 \mathrm{~N}^{\circ}$ 1, pp. 3-27.

Amorim Neto, O. y Strom, Kaare (2006). "Breaking the Parliamentary Chain of Delegation: Presidents, Non-partisan Cabinet Members in European Democracies". Brazilian Journal of Political Science Vol. 36, pp. 619-643.

Andeweg R. y Timmermans, A. (2008). "Conflict Management in Coalition Government". En Strøm, K.; Müller W. y Bergman, T (eds). Cabinets and Coalition Bargaining: the Democratic Life Cycle in Western Europe. Oxford University Press.

Bäck, H.; P. Dumont, H.E. Meier,T. Persson y K.Vernby (2009). “Does European Integration Lead to a 'Presidentialization' of Executive Politics?”. European Union Politics, Vol. 2, $\mathrm{N}^{\circ}$ 2, pp. 226-252.

Blondel, Jean; Ferdinand Müller-Rommel (2001). Cabinets in Eastern Europe. Londres: Palgrave.

Blondel, Jean; Ferdinand Müller-Rommel y Darina Malová (2010). Governing new European democracies. Londres: Palgrave Macmillan.

Boeninger, Edgardo (1998). Democracia en Chile. Lecciones para la gobernabilidad, Santiago: Editorial Andrés Bello. 
Botella, Joan y Rodríguez, Juan (2005). "Reclutamiento y carrera ministerial de los ministros en España (1976 - 2004). VII Congreso de Ciencia Política y de la Administración: Democracia y Buen Gobierno. España.

Botella, Joan; Juan Rodríguez, Oscar Barberá, Astrid Barrio (2011). "Las carreras políticas de los jefes de gobierno regionales en España, Francia y el Reino Unido (1980-2010)”. Revista española de investigaciones sociológicas, $\mathrm{N}^{\circ} 133$, pp. 3-20.

Botero, Felipe (2011). “Carreras políticas en América Latina. Discusión teórica y ajustes de supuestos”. Revista Posdata,Vol. 16, № 2, pp. 167-187.

Browne, Eric y Franklin, Marc (1973). "Aspects of coalition pay offs in European parliamentary democracies". The American Political Science Review.Vol. 67, No 2, pp. 453-469.

Buckley Stephen (2006). The Prime Minister and Cabinet. Edinburgh University Press.

Camerlo, Marcelo (2012). "Political Careers and Minister Turnover under Presidentialism". Paper preparado para el $6^{\circ}$ Congreso Latinoamericano de Ciencia Política, Quito,junio 13-15.

Campus, Donatella (2006). L'antipolitica al governo. Boloña: Il Mulino.

Campus, Donatella (2010). "El lenguaje populista en el poder". Revista de Sociología, No 24, pp. 151-164.

Chasquetti, Daniel (2008). Democracia, presidencialismo y partidos políticos en América Latina: Evaluando la 'difícil combinación'. Montevideo: Instituto de Ciencia Política, Universidad de la República.

Dávila, Mireya (2010). “Tecnocracia y democracia en el Chile contemporáneo: el caso de los gobiernos de la Concertación (1990-2010)”. Revista de Sociología, № 24, pp. 199-217.

Dávila, Mireya (2011). “Tecnocracia y política en el Chile postautoritario (1990-2010), en Alfredo Joignant y Pedro Güell (eds.). Notables, tecnócratas y mandarines. Elementos de sociología de las élites en Chile (1990-2010). Santiago: Ediciones Universidad Diego Portales.

De la Maza, Gonzalo (2011). "Elitismo democrático, líderes civiles y tecnopolítica en la reconfiguración de las élites políticas", Alfredo Joignant y Pedro Güell (eds.). Notables, tecnócratas y mandarines. Elementos de sociología de las élites en Chile (1990-2010), Santiago: Ediciones Universidad Diego Portales.

Dogan, Mattei (1981). "La sélection des ministres en Italie:Dix règles non-écrites" International Political Science Review Vol. 2, Nº 2 pp. 189-210.

Gamson, William (1961). “A theory of coalition formation”. American Sociological Review. Vol. 26. $\mathrm{N}^{\mathrm{o}} 3$, pp. 373-382. 
Garretón, Manuel Antonio (1992). "El Aprendizaje Político en la Transición Chilena”. Documento de Trabajo 24. FLACSO-Chile.

Hidalgo, Paulo (2011). El ciclo político de la Concertación (1990-2010). Santiago: Uqbar.

Huber, John. y Martínez Gallardo, Cecilia (2008). "Replacing Cabinet Ministers: Patterns of Ministerial Stability in Parliamentary Democracies". American Political Science Review Vol. 102, No 2 pp. $169-180$.

Huneeus, Carlos (2000). El régimen de Pinochet, Santiago: Editorial Sudamericana.

Huneeus, Carlos (2005). "Las Coaliciones de Partidos: ¿Un Nuevo Escenario para el Sistema Partidista Chileno?” Revista PolíticaVol. 45, pp. 67-86.

Joignant, Alfredo (2003). "La democracia de la indiferencia. Despolitización, desencanto y malestar en el Gobierno de Eduardo Frei Ruiz-Tagle”, en Óscar Muñoz y Carolina Stefoni (coord.). El período del Presidente Frei Ruiz-Tagle, Santiago:Editorial UniversitariaFlacso.

Joignant, Alfredo (2011). "Tecnócratas, technopols y dirigentes de partido: tipos de agentes y especies de capital en las elites gubernamentales de la Concertación (1990-2010)", en Alfredo Joignant y Pedro Güell (eds.). Notables, tecnócratas y mandarines. Elementos de sociología de las élites en Chile (1990-2010), Santiago: Ediciones Universidad Diego Portales, pp. 49-76.

Lanzaro, Jorge (2003). “Tipos de presidencialismo y modos de gobierno en América Latina”, en Jorge Lanzaro comp.). Tipos de presidencialismo y coaliciones políticas en América Latina, Buenos Aires: Clacso.

Laver Michael (2000). "Government Formation and Public Policy". PS: Political Science and Politics, Vol. 33, N 1, pp. 21-23.

Mardones, Rodrigo (2007). "Chile: todas íbamos a ser reinas”. Revista de Ciencia Política, Vol. 27, pp. 79-96.

Martínez Gallardo, Cecilia (2011). "Out of the Cabinet: What Drives Defections from the Government in Presidential Regimes?" Paper prepared for the ECPR-IPSA Conference. Sao Pablo, Brasil.

Morales, Mauricio (2008). "La primera mujer presidenta de Chile: ¿Qué explica el triunfo de Michelle Bachelet en las elecciones de 2005 a 2006?”. Latin America Research Review, Vol. 43, No 1, pp. 7-32.

Murillo, María Victoria (2001). Labor Unions, Partisan Coalitions, and Market Reforms in Latin America, Cambridge: Cambridge University Press. 
Pérez-Liñán, Aníbal (2009). Juicio político al presidente y nueva inestabilidad política en América Latina, Fondo de Cultura Económica, Buenos Aires.

Reniu, Josep y Albala, Adrián (2010). "Los gobiernos de coalicion y su incidencia sobre los presidencialismos latinoamericanos: el caso del Cono Sur". Working Papers 296. Barcelona, España.

Riker,W. (1962). The Theory of Political Coalitions. New Haven:Yale University Press.

Rodríguez Teruel, Juan (2009). "Reclutamiento y carrera ministerial de los ministros en España (1976-2005)”, Working Paper, № 273, Barcelona: Institut de Ciències Polítiques y Socials.

Siavelis, Peter (1997). "Continuity and Change in the Chilean party system: on the transformational effects of electoral reforms". Comparative Political Studies. Vol. 30. N ${ }^{\circ}$ 6, pp. 651-675.

Silva, Patricio (2010). En el nombre de la razón. Tecnócratas y política en Chile, Santiago: Ediciones Universidad Diego Portales.

Silva, Patricio (2011). "La elite tecnocrática en la era de la Concertación”, en Alfredo Joignant y Pedro Güell (eds.). Notables, tecnócratas y mandarines. Elementos de sociología de las élites en Chile (1990-2010), Santiago: Ediciones Universidad Diego Portales.

Stokes, Susan C. (2001). Mandates and Democracy. Neoliberalism by Surprise in Latin America, Cambridge: Cambridge University Press.

Strøm, Kaare (1990). "A Behavioral Theory of Competitive Political Parties”. American Journal of Political Sciences, Vol. 34, N², pp. 565-598.

Pedro Tavares de Almeida, Antonio Costa Pinto y Nancy Bermeo (eds.) (2003). Who Governs Southern Europe? Regime Change and Ministerial Recruitment, 1850 - 2000. Londres: Frank Cass.

Valdés, Juan Gabriel (1989). La Escuela de Chicago: operación Chile, Buenos Aires: Grupo Editorial Zeta.

Valenzuela, Arturo (1978). El Quiebre de la democracia en Chile. Santiago: FLACSO.

Varas, Augusto (2012). La democracia frente al poder. Santiago: Catalonia.

Weber, Max (1996). El político y el científico. Madrid: Alianza Editorial. 